\title{
Novel variants in DNAH9 lead to nonsyndromic severe asthenozoospermia
}

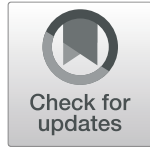

Dongdong Tang ${ }^{1,2,3+} \mathbb{C}_{\text {D }}$, Yanwei Sha ${ }^{4,5+}$, Yang Gao ${ }^{1,2,3 \dagger}$, Jingjing Zhang ${ }^{1 \dagger}$, Huiru Cheng ${ }^{1,2,3}$, Jungiang Zhang ${ }^{1,2,3}$,

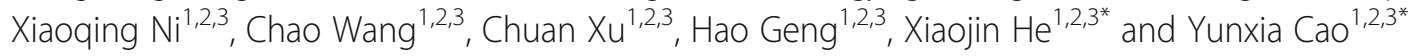

\begin{abstract}
Background: Asthenozoospermia is one of the most common causes of male infertility, and its genetic etiology is poorly understood. DNAH9 is a core component of outer dynein arms in cilia and flagellum. It was reported that variants of DNAH9 (OMIM: 603330) might cause primary ciliary dyskinesia (PCD). However, variants in DNAH9 lead to nonsyndromic severe asthenozoospermia have yet to be reported.

Methods: Whole exome sequencing (WES) was performed for two individuals with nonsyndromic severe asthenozoospermia from two non-consanguineous families, and Sanger sequencing was performed to verify the identified variants and parental origins. Sperm routine analysis, sperm vitality rate and sperm morphology analysis were performed according the WHO guidelines 2010 (5th edition). Transmission electron microscopy (TEM, TECNAI10,80 kV, Philips, Holland) was used to observe ultrastructures of sperm tail. Quantitative realtime-PCR and immunofluorescence staining were performed to detect the expression of DNAH9-mRNA and location of DNAH9protein. Furthermore, assisted reproductive procedures were applied.
\end{abstract}

Results: By WES and Sanger sequencing, compound heterozygous DNAH9 (NM_001372.4) variants were identified in the two individuals with nonsyndromic severe asthenozoospermia (F1 II-1: c.302dupT, p.Leu101fs*47 / c.6956A > G, p.Asp2319Gly; F2 II-1: c.6294 T > A, p.Phe2098Leu / c.10571 T > A, p.Leu3524GIn). Progressive rates less than 1\% with normal sperm morphology rates and normal vitality rates were found in both of the two subjects. No respiratory phenotypes, situs inversus or other malformations were found by detailed medical history, physical examination and lung CT scans etc. Moreover, the expression of DNAH9-mRNA was significantly decreased in sperm from F1 II-1. And expression of DNAH9 is lower in sperm tail by immunofluorescence staining in F1 II-1 compared with normal control. Notably, by intracytoplasmic sperm injection (ICSI), F1 II-1 and his partner successfully achieved clinical pregnancy.

Conclusions: We identified DNAH9 as a novel pathogenic gene for nonsyndromic severe asthenospermia, and ICSI can contribute to favorable pregnancy outcomes for these patients.

Keywords: Asthenozoospermia, Nonsyndromic, Flagellum, DNAH9, ICSI

\footnotetext{
*Correspondence: hxj0117@126.com; caoyunxia6@126.com

${ }^{\dagger}$ Dongdong Tang, Yanwei Sha, Yang Gao and Jingjing Zhang contributed equally to this work.

${ }^{1}$ Reproductive Medicine Center, Department of Obstetrics and Gynecology, the First Affiliated Hospital of Anhui Medical University, No 218 Jixi Road, Hefei 230022, Anhui, China

Full list of author information is available at the end of the article
}

(C) The Author(s). 2021 Open Access This article is licensed under a Creative Commons Attribution 4.0 International License, which permits use, sharing, adaptation, distribution and reproduction in any medium or format, as long as you give appropriate credit to the original author(s) and the source, provide a link to the Creative Commons licence, and indicate if changes were made. The images or other third party material in this article are included in the article's Creative Commons licence, unless indicated otherwise in a credit line to the material. If material is not included in the article's Creative Commons licence and your intended use is not permitted by statutory regulation or exceeds the permitted use, you will need to obtain permission directly from the copyright holder. To view a copy of this licence, visit http://creativecommons.org/licenses/by/4.0/ The Creative Commons Public Domain Dedication waiver (http://creativecommons.org/publicdomain/zero/1.0/) applies to the data made available in this article, unless otherwise stated in a credit line to the data. 


\section{Background}

Infertility is worldwide problem, affecting $8-15 \%$ couples of childbearing age, and male factors might contribute to nearly half of the cases [1-3]. Sufficient sperm motility is very important for sperm entering the female genital tract and oocyte fertilization [4]. According to the WHO guideline (2010), progressive motility $<32 \%$ or total motility $<40 \%$ in at least twice semen analysis at different times were defined as asthenospermia [5]. As one of the most common factors contributing to male infertility, asthenospermia might be associated with some cellular and molecular factors. Firstly, flagella/cilia, which is an important component of sperm tail structure, play a vital role in sperm motility system. Structural defects of flagella/cilia in sperm tail affect sperm motility directly. Secondly, ion channels, especially calcium channel is essential for sperm ion homeostasis, $\mathrm{pH}$ regulation and progressive motility. Thirdly, dysfunction of ATP production in mitochondria is also a principal feature of sperm immobility. Fourthly, some genetic mutations might have negative effects on sperm immobility. Fifthly, lifestyle risk factors, such as smoking, alcohol consumption, chemical pesticides exposure, etc. [6].

Severe asthenozoospermia, which is defined as the proportion of progressive spermatozoa less than $1 \%$, is a tricky problem in infertile men $[7,8]$. Previous studies have presented that genetic disorders might be related to severe asthenospermia. Sha et al. has found that EIF4G1 (OMIM: 600495) was a candidate gene of severe asthenospermia in one patient, whose sperm was revealed with mitochondrial sheath defects [9]. Similarly, $\mathrm{Xu}$ et al. identified a homozygous SPAG17 (OMIM: 616554) mutation was associated with severe asthenozoospermia in a familial twins [10]. Additionally, AKAP3 (OMIM: 604689), AKAP4 (OMIM: 300185), DNAH1 (OMIM: 603332), CATSPER2 (OMIM: 607249) and NSUN7 (OMIM: 6171855), etc. were also revealed to be associated with severe asthenozoospermia [11-15].

Furthermore, severe asthenospermia is also revealed as one of the representative features of primary ciliary dyskinesia (PCD, OMIM: 244400), which includes a wide motile dysfunction of cilia in respiratory tract and sperm, etc. Most of the PCD cases are associated with genetic mutations, and some genes have been identified, such as DNAH5 (OMIM: 603335), DNAH9 (OMIM: 603330) and DNAH11 (OMIM: 603339), etc. [16-18]. DNAH9 encodes a part of outer dynein arm (ODA) heavy chains components, and expressed on the entire length of the sperm tail [16]. Several researches have reported that mutations in DNAH9 caused PCD [19, 20]. Fassad et al. conducted a study including 536 patients with PCD, and 4 cases from 3 families were found carrying DNAH9 loss-of-function mutations. One of the four cases was presented with marked asthenospermia, and other 3 cases were not tested fertility due to too young age [19]. Loges et al. investigated 548 individuals with classical PCD symptoms or suspected PCD, and found 5 cases with mutations in DNAH9. However, no spermrelated examinations were performed in these cases [20].

Although mutations in DNAH9 have been identified to be an important cause of syndromic asthenospermia, such as PCD, the association between DNAH9 and nonsyndromic asthenospermia has not been examined. And whether DNAH9 variants have effects on structures and ultrastructures of sperm tail, remain unclear. In this study, whole exome sequencing (WES) was performed in two Chinese men with severe nonsyndromic asthenospermia, and compound heterozygous variants in DNAH9 were identified in these two cases.

\section{Methods}

\section{Subjects}

Two Han Chinese patients, presenting to the Reproductive Center of the First Affiliated Hospital of Anhui Medical University (Hefei, China) and the Xiamen Maternity and Child Care Hospital (Xiamen, China) respectively, and diagnosed with severe asthenospermia were enrolled in this study. Both subjects met several criteria as follows: without obvious PCD-related symptoms, such as chronic cough, recurrent infections of airways, as well as situs inversus and laterality disorders, etc.; with normal somatic karyotypes (46, XY); with normal semen volume $(\geq 1.5 \mathrm{ml})$, sperm concentration $\left(\geq 15 \times 10^{6} / \mathrm{ml}\right)$, total sperm count $\left(\geq 39 \times 10^{6} / \mathrm{ml}\right)$; with a proportion of progressive spermatozoa $<1 \%$; with a proportion of normal sperm morphology $\geq 4 \%$; with normal sperm vitality rates $(\geq 58 \%)$. Two subjects with normal fertility and normal semen characteristics were enrolled as control group. Peripheral whole blood of all cases was collected.

\section{Ethical approval}

This study was approved by the Ethics Committee of the First Affiliated Hospital of Anhui Medical University and the Ethics Committee of Xiamen Maternity and Child Care Hospital. All the individuals and their family members, as well as two controls signed written informed consents after having received complete information about the research.

\section{Sperm analysis}

Sperm routine analysis, sperm vitality rate and sperm morphology analysis were performed according the WHO guidelines 2010 (5th edition) [5].

\section{WES, bioinformatic analysis and sanger sequencing}

DNA from whole peripheral blood was used to perform WES and bioinformatic analysis. And the protocols were in accordance with previous research [9, 21]. Sanger 
sequencing was performed to verify the identified variants and parental origins. The primers are listed in Supplementary Table 1.

\section{Transmission electron microscopy}

Sperm sample was prepared and treated as described previously $[9,21]$. Subsequently, transmission electron microscopy (TEM, TECNAI-10, 80 kV, Philips, Holland) was used to observe ultrastructures of sperm tail.

\section{Quantitative real-time PCR (QRT-PCR) and} immunofluorescence staining

QRT-PCR experiment of DNAH9-mRNA and immunofluorescence staining experiment of DNAH9-protein in sperm were performed in accordance with previous study $[9,21]$. The primary anti-DNAH9 was Anti-Dynein heavy chain antibody (ab133968, ABCAM). And the PCR primers were presented in Supplementary Table 2.

\section{Assisted reproductive procedures}

The spouse of F1 II-1 underwent standard controlled ovarian hyperstimulation, and oocyte retrieval. Very few slightly motile spermatozoa were used for subsequent intracytoplasmic sperm injection (ICSI). The embryos were cultured to day 5 or day 6 . Two viable thawed embryos were transferred 2 months later. Clinical pregnancy was confirmed by ultrasound performed 28 days after embryo transfer.

\section{Results}

Compound heterozygous variants in DNAH9 were identified in the individuals with severe asthenozoospermia

To detect the cause of severe asthenozoospermia in these two patients, WES analysis was performed. Bioinformatical analysis were applied to seek for meaningful homozygous or compound heterozygous variants. Potentially pathogenic variants were obtained according to criteria as follows: allele frequencies $<1 \%$ in the ExAc_all, $1 \mathrm{KGP}$ and gnomAD databases; potentially high pathogenicity predicted by Sorting Intolerant From Tolerant (SIFT), PolyPhen-2, and Mutation Taster; extremely high expression in the testis. After the bioinformatical analysis, both of the two cases were found to carry compound heterozygous variants in DNAH9, the only gene associated with spermatozoa activity in genes met the above criteria. Subsequently, validation of Sanger sequencing for DNAH9 was performed in the two cases and their parents. Compound heterozygous variants in DNAH9 were validated in these two patients, and their parents were determined to be heterozygous carriers. The detailed information were presented in Table 1, Fig. 1 and Supplementary Fig. 1. The predicted part three-dimensional structure of mutated DNAH9 residues were presented in Supplementary Fig. 2 by SWISSMODEL software (https://swissmodel.expasy.org/).

\section{Sperm analysis and PCD-related symptoms of the two individuals carrying DNAH9 compound heterozygous variants}

The clinical features and results of sperm tests were presented in Table 2. Both of the patients were found with proportions of progressive spermatozoa less than $1 \%$, normal sperm vitality, and normal morphological sperm rates. No significant defects in sperm tail were found by Papanicolaou Staining method. Additionally, no PCD-related symptoms, such as chronic cough, recurrent infections of airways, as well as situs inversus and laterality disorders, etc., were found in these two cases. And the CT lung screening showed a normal result in the F1 II-1 (Supplementary Fig. 3). To further exclude possible ultrastructural defects, we performed TEM for spermatozoa from F1 II-1.

Table 1 Genetic information of DNAH9 mutations of the two individuals

\begin{tabular}{|c|c|c|c|c|}
\hline Individual & F1 II-1 & & F2 II-1 & \\
\hline cDNA mutation & c.302dupT & c.6956A > G & c.6294 T > A & c. $10571 \mathrm{~T}>\mathrm{A}$ \\
\hline Mutation type & frameshift & nonsynonymous & nonsynonymous & nonsynonymous \\
\hline Protein alteration & p.Leu101fs*47 & p.Asp2319Gly & p.Phe2098Leu & p.Leu3524Gln \\
\hline \multicolumn{5}{|c|}{ Allele frequency in human population } \\
\hline $1 \mathrm{KGP}$ & 0 & 0 & 0 & 0 \\
\hline ExAc_all & 0.0008 & 0.00002 & 0 & 0 \\
\hline gnomAD & 0.0003 & 0.00002 & 0 & 0 \\
\hline \multicolumn{5}{|c|}{ Functional prediction } \\
\hline SIFT & N/A & D & D & $\mathrm{D}$ \\
\hline PolyPhen-2 & N/A & D & $\mathrm{D}$ & $\mathrm{D}$ \\
\hline MutationTaster & N/A & $D$ & $D$ & $\mathrm{D}$ \\
\hline
\end{tabular}

RefSeq accession number of DNAH9 is NM_001372.4

Abbreviations: 1KGP 1000 Genomes Project, ExAc_all all the data of Exome Aggregation Consortium, gnomAD the Genome Aggregation Database, N/A not applicable, $D$ Disease-causing 
A

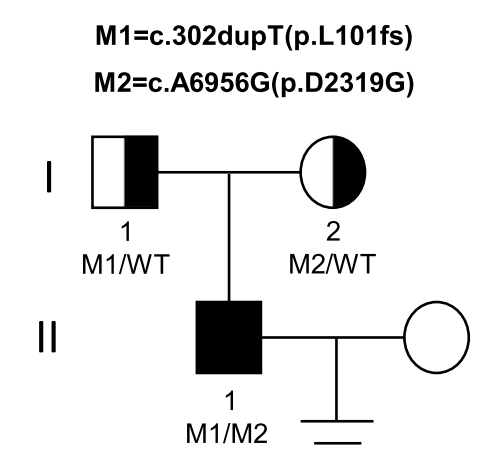

M1 C C

T G T T C G A C A A G T A G

M2

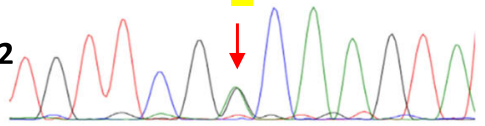

B

Family 2

$M 3=c . T 6294 A(p . F 2098 L)$

$M 4=c . T 10571 A(p . L 3524 Q)$

I

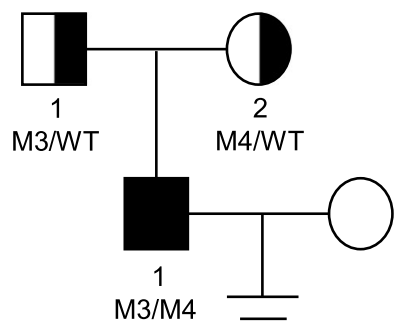

$C \subset T C T T T C C C G C C$

M3

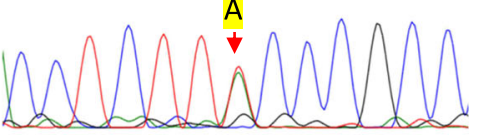

G A C C C C T G C T T G G

A

M4

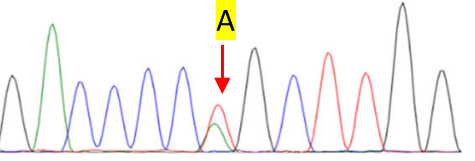

C

DNAH9 Protein: NP_001363.2

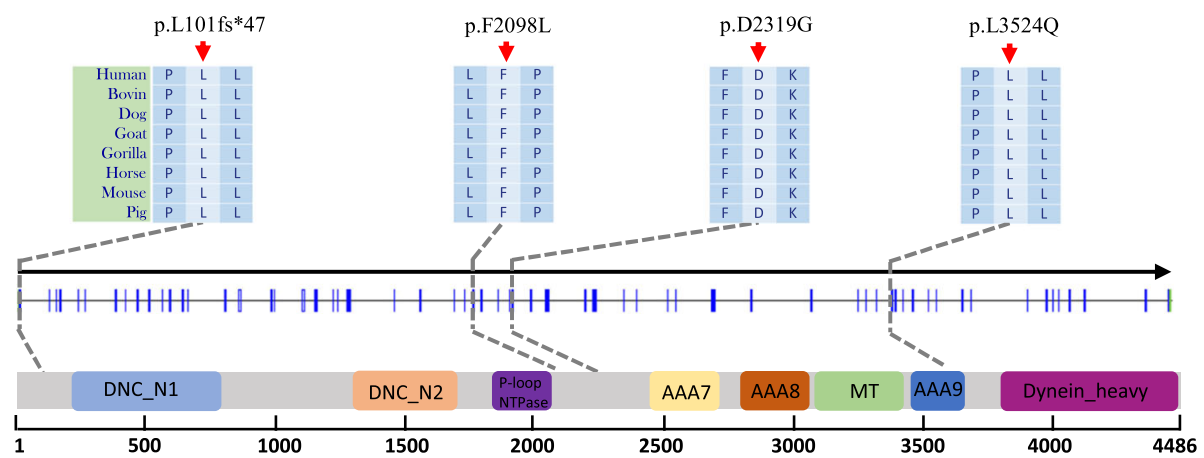

Fig. 1 Variants of DNAH9 in the two patients. $\mathbf{a}-\mathbf{b}$ The two families affected by the variants in DNAH9. The red arrows indicate mutated positions in the Sanger sequencing results. c The mutated positions of DNAH9 are conserved among species. And the red arrows indicate the locations of DNAH9 variants occurred in the domains of DNAH9 protein. $\mathrm{M}$, DNAH9 variant; $\mathrm{WT}$, wild type

No obvious defects were found in mitochondrial sheath and flagellum in F1 II-1 compared with normal control. The typical " $9+2$ " microtubule structure were found in spermatozoa from both F1 II-1 and the control. Similarly, the typical structure of ODA was present in F1 II-1 and control (Supplementary Fig. 4).

\section{Expression of DNAH9 was decreased in spermatozoa from} F1 II-1

As seminal fluid of F2 II-1 was not obtained, only spermatozoa from F1 II-1 were examined to determine the potential effect of variants on $D N A H 9$. In the healthy control sperm, DNAH9 is highly expressed in sperm tail by immunofluorescence staining. However, expression of DNAH9 is lower in sperm of F1 II-1. In order to determine expressive level of mRNA of DNAH9, QRT-PCR was performed. And it was found that expressive level of DNAH9-mRNA in F1 II-1 was significantly lower than normal control (Fig. 2).

Pregnancy outcome of patient with DNAH9 variants Standard controlled ovarian hyperstimulation, oocyte retrieval, and ICSI was performed for wife of F1 II-1. Sixteen oocytes in MII stage were retrieved. All the eggs 
Table 2 Clinical Features of the Two Individuals Carrying DNAH9 Mutations

\begin{tabular}{|c|c|c|c|c|}
\hline Individual & F1 II-1 & & F2 II-1 & \\
\hline Age & 29 & & 28 & \\
\hline Semen analysis & Sample 1 & Sample 2 & Sample 1 & Sample 2 \\
\hline Semen volume $(\mathrm{mL})$ & 4.2 & 5.2 & 2.8 & 3.2 \\
\hline Sperm concentration $\left(10^{6} / \mathrm{mL}\right)$ & 133.7 & 55.3 & 78.6 & 43.8 \\
\hline Progressive motility (\%) & 0.3 & 0.8 & 1.0 & 0.5 \\
\hline Normal morphology (\%) & 6.5 & 5 & 4 & 4.5 \\
\hline Sperm vitality (\%) & 75 & 82 & 68 & 72 \\
\hline DFI (\%) & 10.18 & N/A & N/A & N/A \\
\hline \multicolumn{5}{|l|}{ PCD-related phenomenon } \\
\hline Rhinosinusitis & No & & No & \\
\hline Wet cough & No & & No & \\
\hline Otitis media & No & & No & \\
\hline Bronchiectasis & No & & No & \\
\hline Situs inversus & No & & No & \\
\hline Congenital heart disease & No & & No & \\
\hline
\end{tabular}

Abbreviations: DFI DNA fragmentation index, N/A Not applicable, $P C D$ Primary ciliary dyskinesia

Bold characters indicate abnormal values

got fertilization, and 11 fertilized eggs reached D5/D6 embryos. Subsequently, two high-quality D5 embryos were transferred after two-month frozen. The hCG level was $1300 \mathrm{mIU} / \mathrm{ml}$ in the blood 14 days after transfer, and clinical pregnancy was verified by ultrasound performed 28 days after embryo transfer (Fig. 3).

\section{Discussion}

In this present study, we found two patients with severe asthenozoospermia harboring compound heterozygous variants in DNAH9. And the expression of DNAH9 was significant decreased in one patient with DNAH9 variants, although no obvious morphological and ultrastructural defects were found. Moreover, no other PCD-related symptoms were found in these two cases. These results presented that DNAH9 variant is a novel causative genetic etiology of nonsyndromic severe asthenozoospermia.

DNAH9, mapping to $17 \mathrm{p} 12$, is a human axonemal dynein beta heavy chain gene. There are 69 exons in this $14 \mathrm{~kb}$-long cDNA of DNAH9, which encode a protein as a core component of outer dynein arms in cilia and flagella [22]. As cilia or flagella are highly conserved in varieties of human cells and organs, the mutations of DNAH9 may be associated with multisystemic disorders, including bronchiectasis, recurrent respiratory tract infections, and male infertility, etc. $[16,19,20,23]$. In the study conducted by Fassad et al., all the four patients carrying DNAH9 mutations had syndromic clinical features, such as rhinosinusitis, wet cough and situs inversus, etc. [19]. Similarly, Loges et al. also found that respiratory phenotype or laterality defect in all the five individuals harboring DNAH9 mutations. However, only one patient with DNAH9 mutation was described with asthenospermia [20]. In our research, two cases carrying DNAH9 compound heterozygous variants only presented with severe asthenospermia, without any respiratory symptoms or laterality defect by systemic medical history and physical examination. As no PCD-related symptoms in both cases and F2 II-1 losing follow-up, some invasive procedures, such as respiratory ciliary biopsy, etc. were not performed to test morphology or ultrastructure of other ciliary. Nonetheless, it was speculated that at least sperm motile defect was present in these patients with DNAH9 compound heterozygous variants. The pathogenic DNAH9 variants lead to significant decreased DNAH9 at mRNA and protein levels, however, we failed to observe obvious ultrastructure defects in sperm of F1 II-1. This could be due to the complexity of ODA, which composed of several heavy chain proteins, including DNAH5, DNAH8, DNAH11, DNAH17, et al. [19, 20, 24, 25]. These normal proteins can form a seemingly normal ODA structure, however, its dynamic ability damaged dramatically, causing severe asthenozoospermia. This in turn validated the essential role of DNAH9 in ODA function.

The diverse types of the variants in DNAH9 might lead to different disease phenotypes and varying ciliary defects. The clinical phenotype in these two patients are less severe compared with other individuals harboring DNAH9 mutations with classical PCD symptoms. The identified compound heterozygous 
A

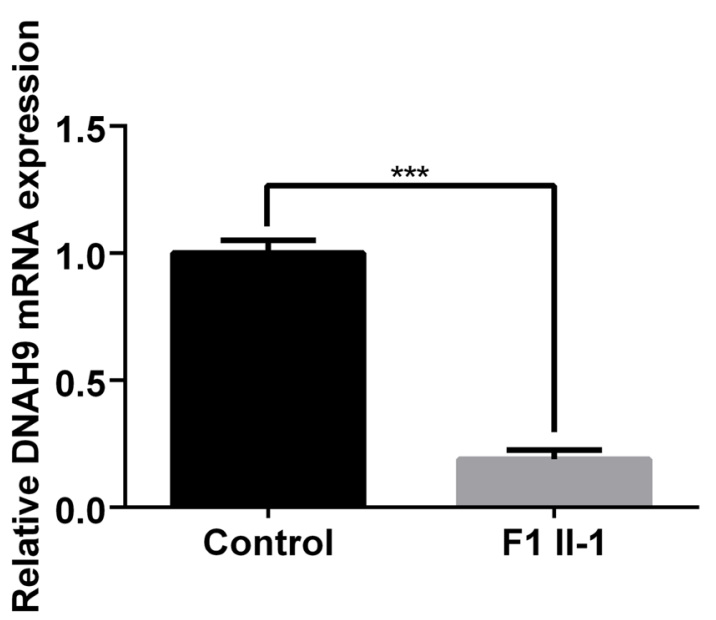

B

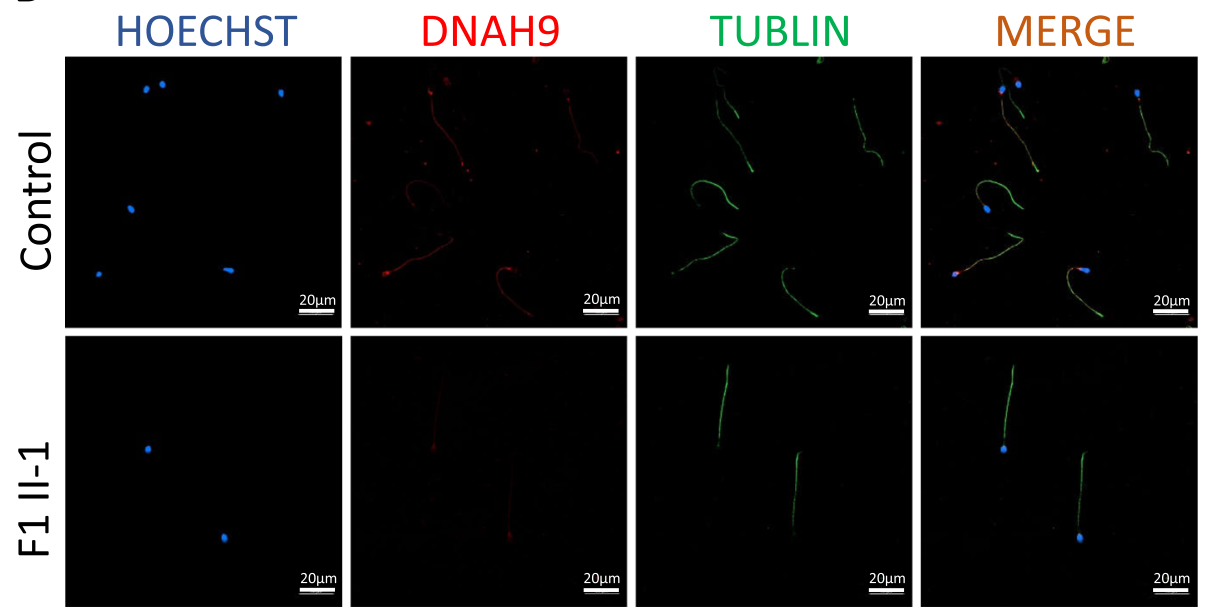

Fig. 2 a The expression of DNAH9-mRNA in spermatozoon is significantly lower in F1 II-1 compared with normal control. b Immunofluorescence staining in spermatozoon from the DNAH9-mutated F1 II-1 and normal control. DNAH9 immunostaining (red) was primarily concentrated at the sperm neck and flagellum in a punctate pattern, while the immunostaining was decreased in the sperm of F1 II-1. Hoechst (blue) was stained as a nuclear marker. The anti-tubulin (green) was stained as a marker of flagellum

variants in these two cases both includes missense variants, milder genetic defects, which might have only mild harmful effects on structure and function of DNAH9. Therefore, only severe sperm motility defects were presented in these two cases. It was partially supported by the normal morphological and ultrastructure in sperm of F1 II-1. Unlike cases in the studies of Fassad and Loges et al., no laterality defects were found in these two patients. DNAH9 is an important core of ODA, whose mutations may result in impaired ciliary motility and subsequent impaired or disturbed leftward fluid flow within the embryonic node. Therefore, only nearly half of patients with PCD present situs inversus. Additionally, the milder genetic defects of DNAH9 in these two cases may had little effects on the fluid flow within the embryonic node. These factors may contribute to the normal laterality in these two patients. Despite of DNAH9, diverse variants of some other candidate genes of PCD could also lead to various phenotypes. $D N A H 1$, a core component of inner-arm heavy chain dynein, was also considered as a candidate gene causing PCD [26, 27]. Similarly, twelve patients with DNAH1 variants were only exhibited multiple morphologic abnormalities of the flagella (MMAF) phenotype, but without PCD in the study conducted by Sha et al. [28].

Severe asthenozoospermia is a rare disease, and assisted reproduction techniques (ART), especially ICSI is an important and even sole method for these patients to get pregnant. The wife of F1 II-1 also received the examination of DNAH9 gene, and no abnormal variants were found. Considering an autosomal recessive pattern of inheritance, the F1 II-1 couple underwent standard 


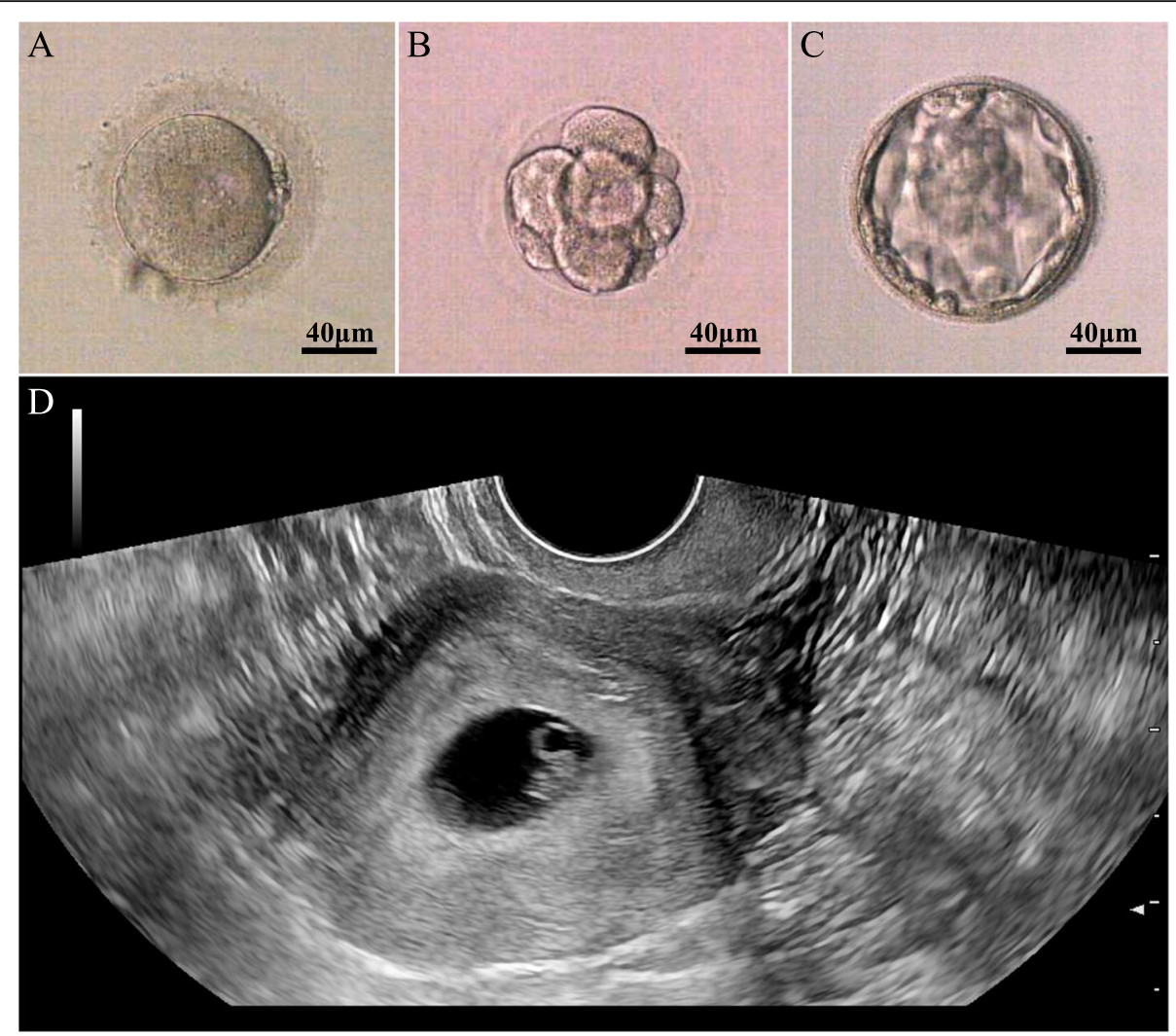

Fig. 3 Typical morphology of the implanted embryo from F1 II-1. Well quality blastocysts formed after standard embryo culture and 2 embryos were transferred into the partner's uterus. The embryo was implanted and yielded clinical pregnancy. a: 2 pronuclear (PN) fertilization, (b): 8-cell stage embryo, (c): blastocyst stage embryo.(d): the ultrasonography of gestational sac. Scale bar:40 $\mu \mathrm{m}$

ICSI procedure, and get good clinical outcome. It is also the first time to report that ICSI can be recommended for severe asthenozoospermia caused by DNAH9 variants.

Some limitations should also be taken into consideration. Firstly, the sperm sample of F2 II-1 can not be obtained. So all the experiments of sperm were only performed in F1 II-1. Additionally, respiratory ciliary was not collected for further experiments as a invasive procedure. Secondly, as the not enough sperm sample, western blot experiment was not performed to examine the protein expressive level of DNAH9 in F1 II-1. Thirdly, we did not explore the prevalence of DNAH9 variant in large-scale population with nonsyndromic severe asthenospermia, which will be an important work in future.

\section{Conclusions}

In summary, variants of DNAH9 are novel pathogenic factors for not only syndromic severe asthenospermia, such as PCD, but also nonsyndromic severe asthenospermia. ICSI can be recommended as a first-line treatment for favorable pregnancy outcomes for these patients.

\section{Supplementary Information}

The online version contains supplementary material available at https://doi. org/10.1186/s12958-021-00709-0.

Additional file 1: Supplementary Table 1. Primers used for verification of DNAH9 mutations.

Additional file 2: Supplementary Table 2. Primers used for QRT-PCR assay of DNAH9 and $\beta$-actin.

Additional file 3: Supplementary Fig. 1. Sanger sequencing results of of two cases and their parents.

Additional file 4: Supplementary Fig. 2. The predicted part threedimensional structure of mutated DNAH9 residues by SWISS-MODEL software (https://swissmodel.expasy.org/); WT, wild type.

Additional file 5: Supplementary Fig. 3. Diagnostic imaging tests excluded typical PCD signs. (A): The chest $X$ rays showed the heart on the left. (B): The chest $C T$ showed normal pulmonary bronchus. (C): The upper abdomen $C T$ showed regular visceral structure.

Additional file 6: Supplementary Fig. 4. Sperm morphology and ultrastructure in the F1 II-1 with DNAH9 compound heterozygous variants. (A-D) Normal spermatozoon from a healthy control man with normal fertility. (E-H) Spermatozoon from F1 II-1 with severe asthenospermia. Sperm morphology analysis showed normal long flagella in the control man (A), and F1 II-1 (E). TEM showed the typical " $9+2$ " microtubule structure as well as normal outer dynein arms in spermatozoa of the control man (B$D)$, and F1 II-1 (F-H). Scale bars: $10 \mu \mathrm{m}$ in (A) and (E); 1 um in (B-D) and (FH). CP, central pair of microtubules; PM, peripheral microtubule doublets; ODF, outer dense fiber; MS, mitochondrial sheath; TEM, transmission electron microscopy. 


\section{Abbreviations}

PCD: Primary ciliary dyskinesia; WES: Whole exome sequencing; TEM: Transmission electron microscopy; ICSI: Intracytoplasmic sperm injection; ODA: Outer dynein arm; QRT-PCR: Quantitative real-time PCR; SIFT: Sorting Intolerant From Tolerant; MMAF: Multiple morphologic abnormalities of the flagella; ART: Assisted reproduction techniques

\section{Acknowledgements}

Not applicable.

\section{Authors' contributions}

YC, XH, DT and YS designed the study. DT, YS, CX and HG collected the data. YG, and JZ analyzed the data of WES and Sanger sequencing. DT, HC, JZ and $X N$ performed the experiments of QRT-PCR and immunofluorescence staining. DT, YS and YG wrote the paper. All authors read and approved the final manuscript.

\section{Funding}

This study was supported by the National Key R\&D Program of China (No. 2019YFC1005106), Natural Science Foundation of Anhui Province in China (No. 1908085QH355) and the National Natural Science Foundation of China (No. 81901541).

\section{Availability of data and materials}

The datasets used and/or analysed during the current study are available from the corresponding author on reasonable request.

\section{Ethics approval and consent to participate}

This study was approved and consented by the Ethics Committee of the First Affiliated Hospital of Anhui Medical University and the Ethics Committee of Xiamen Maternity and Child Care Hospital.

\section{Consent for publication}

All the individuals and their family members, as well as two controls signed written informed consents after having received complete information about the research.

\section{Competing interests}

The authors declare that they have no competing interests.

\section{Author details}

'Reproductive Medicine Center, Department of Obstetrics and Gynecology, the First Affiliated Hospital of Anhui Medical University, No 218 Jixi Road, Hefei 230022, Anhui, China. ${ }^{2} \mathrm{NHC}$ Key Laboratory of study on abnormal gametes and reproductive tract (Anhui Medical University), No 81 Meishan Road, Hefei 230032, Anhui, China. ${ }^{3}$ Key Laboratory of Population Health Across Life Cycle (Anhui Medical University), Ministry of Education of the People's Republic of China, No 81 Meishan Road, Hefei 230032, Anhui, China. ${ }^{4}$ Department of Andrology, United Diagnostic and Research Center for Clinical Genetics, School of Public Health \& Women and Children's Hospital, Xiamen University, Xiamen 361005, Fujian, China. ${ }^{5}$ State Key Laboratory of Molecular Vaccinology and Molecular Diagnostics \& Center for Molecular Imaging and Translational Medicine, School of Public Health, Xiamen University, Xiamen 361102, China.

Received: 20 July 2020 Accepted: 12 February 2021

Published online: 20 February 2021

\section{References}

1. Vander Borght $M$, Wyns C. Fertility and infertility: defifinition and epidemiology. Clin Biochem. 2018;62:2-10

2. Deshpande PS. Causes and prevalence of factors causing infertility in a public health facility. J Hum Reprod Sci. 2019;12:287-93.

3. Sun $\mathrm{H}$, Gong $T$, Jiang YT, Zhang S, Zhao YH, Wu QJ. Global, regional, and national prevalence and disability-adjusted life-years for infertility in 195 countries and territories, 1990-2017: results from a global burden of disease study, 2017. Aging (Albany NY). 2019;11:10952-91.

4. Shin TE, Park JW, Jeon WY, Lee EJ, Kwon H, Jeon B, Kang HE, Kim MJ, Kim DK, Kim HH, Ko JJ, Lee JH. Motility enhancement of human spermatozoa using electrical stimulation in the nano-ampere range with enzymatic biofuel cells. PLoS One. 2020;15:e0228097.
5. WHO. Examination and processing human semen. 5th ed. New York: Cambridge University Press; 2010

6. Shahrokhi SZ, Salehi P, Alyasin A, Taghiyar S, Deemeh MR. Asthenozoospermia: cellular and molecular contributing factors and treatment strategies. Andrologia. 2020;52:e13463.

7. Al-Malki AH, Alrabeeah K, Mondou E, Brochu-Lafontaine V, Phillips S, Zini A. Testicular sperm aspiration (TESA) for infertile couples with severe or complete asthenozoospermia. Andrology. 2017;5:226-31.

8. Casper RF, Meriano JS, Jarvi KA, Cowan L, Lucato ML. The hypo-osmotic swelling test for selection of viable sperm for intracytoplasmic sperm injection in men with complete asthenozoospermia. Fertil Steril. 1996;65:972-6.

9. Sha Y, Liu W, Huang X, Li Y, Ji Z, Mei L, Lin S, Kong S, Lu J, Kong L, Zhu X, Lu Z, Ding L. EIF4G1 is a novel candidate gene associated with severe asthenozoospermia. Mol Genet Genomic Med. 2019;7:e807.

10. Xu X, Sha YW, Mei LB, Ji ZY, Qiu PP, Ji H, Li P, Wang T, Li L. A familial study of twins with severe asthenozoospermia identified a homozygous SPAG17 mutation by whole-exome sequencing. Clin Genet. 2018;93:345-9.

11. Baccetti B, Collodel G, Estenoz M, Manca D, Moretti E, Piomboni P. Gene deletions in an infertile man with sperm fibrous sheath dysplasia. Hum Reprod. 2005;20:2790-4.

12. Amiri-Yekta A, Coutton C, Kherraf ZE, Karaouzène T, Le Tanno P, Sanati MH, Sabbaghian M, Almadani N, Sadighi Gilani MA, Hosseini SH, Bahrami S, Daneshipour A, Bini M, Arnoult C, Colombo R, Gourabi H, Ray PF. Wholeexome sequencing of familial cases of multiple morphological abnormalities of the sperm flagella (MMAF) reveals new DNAH1 mutations. Hum Reprod. 2016;31:2872-80.

13. Ben Khelifa M, Coutton C, Zouari R, Karaouzène T, Rendu J, Bidart M, Yassine S, Pierre V, Delaroche J, Hennebica S, Grunwald D, Escalier D, Pernet-Gallay K, Jouk PS, Thierry-Mieg N, Touré A, Arnoult C, Ray PF. Mutations in DNAH1, which encodes an inner arm heavy chain dynein, lead to male infertility from multiple morphological abnormalities of the sperm flagella. Am J Hum Genet. 2014;94:95-104.

14. Zhang Y, Malekpour M, Al-Madani N, Kahrizi K, Zanganeh M, Lohr NJ, Mohseni M, Mojahedi F, Daneshi A, Najmabadi H, Smith RJ. Sensorineural deafness and male infertility: a contiguous gene deletion syndrome. J Med Genet. 2007:44:233-40.

15. Khosronezhad N, Colagar AH, Jorsarayi SG. T26248G-transversion mutation in exon7 of the putative methyltransferase Nsun7 gene causes a change in protein folding associated with reduced sperm motility in asthenospermic men. Reprod Fertil Dev. 2015;27:471-80.

16. Fliegauf $\mathrm{M}$, Olbrich $\mathrm{H}$, Horvath J, Wildhaber JH, Zariwala MA, Kennedy M, Knowles MR, Omran H. Mislocalization of DNAH5 and DNAH9 in respiratory cells from patients with primary ciliary dyskinesia. Am J Respir Crit Care Med. 2005;171:1343-9.

17. Lucas JS, Barbato A, Collins SA, Goutaki M, Behan L, Caudri D, Dell S, Eber E, Escudier E, Hirst RA, Hogg C, Jorissen M, Latzin P, Legendre M, Leigh MW, Midulla F, Nielsen KG, Omran H, Papon JF, Pohunek P, Redfern B, Rigau D, Rindlisbacher B, Santamaria F, Shoemark A, Snijders D, Tonia T, Titieni A, Walker WT, Werner C, Bush A, Kuehni CE. European Respiratory Society guidelines for the diagnosis of primary ciliary dyskinesia. Eur Respir J. 2017; 49:1601090.

18. Chioccioli M, Feriani L, Nguyen Q, Kotar J, Dell SD, Mennella V, Amirav I, Cicuta P. Quantitative high-speed video profiling discriminates between DNAH11 and HYDIN variants of primary Ciliary dyskinesia. Am J Respir Crit Care Med. 2019:199:1436-8.

19. Fassad MR, Shoemark A, Legendre M, Hirst RA, Koll F, le Borgne P, Louis B, Daudvohra F, Patel MP, Thomas L, Dixon M, Burgoyne T, Hayes J, Nicholson AG, Cullup T, Jenkins L, Carr SB, Aurora P, Lemullois M, Aubusson-Fleury A, Papon JF, O'Callaghan C, Amselem S, Hogg C, Escudier E, Tassin AM, Mitchison HM. Mutations in outer dynein arm heavy chain DNAH9 cause motile cilia defects and Situs Inversus. Am J Hum Genet. 2018;103:984-94.

20. Loges NT, Antony D, Maver A, Deardorff MA, Güleç EY, Gezdirici A, NötheMenchen T, Höben IM, Jelten L, Frank D, Werner C, Tebbe J, Wu K, Goldmuntz E, Čuturilo G, Krock B, Ritter A, Hjeij R, Bakey Z, Pennekamp P, Dworniczak B, Brunner H, Peterlin B, Tanidir C, Olbrich H, Omran H, Schmidts M. Recessive DNAH9 loss-of-function mutations cause laterality defects and subtle respiratory Ciliary-beating defects. Am J Hum Genet. 2018;103:995-1008.

21. Ni X, Wang J, Lv M, Liu C, Zhong Y, Tian S, Wu H, Cheng H, Gao Y, Tan Q, Chen B, Li Q, Song B, Wei Z, Zhou P, He X, Zhang F, Cao Y. A novel homozygous mutation in WDR19 induces disorganization of microtubules 
in sperm flagella and nonsyndromic asthenoteratospermia. J Assist Reprod Genet. 2020;37:1431-9.

22. Bartoloni L, Blouin JL, Maiti AK, Sainsbury A, Rossier C, Gehrig C, She JX, Marron MP, Lander ES, Meeks M, Chung E, Armengot M, Jorissen M, Scott HS, DelozierBlanchet CD, Gardiner RM, Antonarakis SE. Axonemal beta heavy chain dynein DNAH9: CDNA sequence, genomic structure, and investigation of its role in primary ciliary dyskinesia. Genomics. 2001;72:21-33.

23. Reiter JF, Leroux MR. Genes and molecular pathways underpinning ciliopathies. Nat Rev Mol Cell Biol. 2017;18:533-47.

24. Liu C, Miyata H, Gao Y, Sha Y, Tang S, Xu Z, Whitfield M, Patrat C, Wu H, Dulioust E, Tian S, Shimada K, Cong J, Noda T, Li H, Morohoshi A, Cazin C, Kherraf ZE, Arnoult C, Jin L, He X, Ray PF, Cao Y, Touré A, Zhang F, lkawa M. Biallelic DNAH8 variants Lead to multiple morphological abnormalities of the sperm flagella and primary male infertility. Am J Hum Genet. 2020;107:330-41.

25. Sironen A, Shoemark A, Patel M, Loebinger MR, Mitchison HM. Sperm defects in primary ciliary dyskinesia and related causes of male infertility. Cell Mol Life Sci. 2020;77:2029-48.

26. Emiralioğlu N, Taşkıran EZ, Koşukcu C, Bilgiç E, Atilla P, Kaya B, Günaydın Ö Yüzbaşıŏlu A, Tuğcu GD, Ademhan D, Erylımaz Polat S, Gharibzadeh Hızal M, Yalçın E, Doğru D, Kiper N, Alikaşifoğlu M, Özçelik U. Genotype and phenotype evaluation of patients with primary ciliary dyskinesia: first results from Turkey. Pediatr Pulmonol. 2020:55:383-93.

27. Imtiaz F, Allam R, Ramzan K, Al-Sayed M. Variation in DNAH1 may contribute to primary ciliary dyskinesia. BMC Med Genet. 2015;16:14.

28. Sha Y, Yang $X$, Mei L, Ji Z, Wang X, Ding L, Li P, Yang S. DNAH1 gene mutations and their potential association with dysplasia of the sperm fibrous sheath and infertility in the Han Chinese population. Fertil Steril. 2017:107:1312-8

\section{Publisher's Note}

Springer Nature remains neutral with regard to jurisdictional claims in published maps and institutional affiliations.

Ready to submit your research? Choose BMC and benefit from:

- fast, convenient online submission

- thorough peer review by experienced researchers in your field

- rapid publication on acceptance

- support for research data, including large and complex data types

- gold Open Access which fosters wider collaboration and increased citations

- maximum visibility for your research: over $100 \mathrm{M}$ website views per year

At BMC, research is always in progress.

Learn more biomedcentral.com/submissions 